\title{
Modelling Surgical Cuts, Retractions, and Resections via Extended Finite Element Method
}

\author{
Lara M. Vigneron ${ }^{1}$, Jacques G. Verly ${ }^{1}$, and Simon K. Warfield ${ }^{2}$ \\ 1 Signal Processing Group, Dept. of Electrical Engineering and Computer Science, \\ University of Liège, Belgium \\ 2 Computational Radiology Laboratory, Surgical Planning Laboratory, \\ Brigham and Women's Hospital, Harvard Medical School, Boston, USA
}

\begin{abstract}
We introduce a new, efficient approach for modelling the deformation of organs following surgical cuts, retractions, and resections. It uses the extended finite element method (XFEM), recently developed in "fracture mechanics" for dealing with cracks in mechanical parts. XFEM eliminates the computationally-expensive remeshing that would be required if the standard finite element method (FEM) was used. We report on the successful application of the method to the simulation of $2 \mathrm{D}$ retraction. The method may have significant impact on surgical simulators and navigators.
\end{abstract}

\section{Introduction}

Image-guided surgical navigation systems allow the surgeon to follow more precisely his planning by displaying the positions of surgical instruments in preoperative images. However, as surgery progresses, these images become inaccurate due to the deformations of organs. Even though intraoperative images can be acquired, they have limited signal-to-noise ratio and spatial resolution. Additionally, not all imaging modalities (particularly functional ones) are available intraoperatively. Therefore, it is critical to continue using all preoperative images and to update them as organs deform.

Several nonrigid registration techniques could potentially be used for updating preoperative images. One approach is to model mechanical organ behavior based on the finite element method (FEM), as explained in [4] 5] for the case of the brain. The idea is to capture the displacement of the surface(s) defining the shape of the organ and to compute the resulting deformation of a volume mesh of this organ by linear elastic finite element (FE) calculations.

Most studies of organ deformation based on biomechanical models have focused on the early stages of surgery, i.e., prior to significant deformations and any cut. The precision achieved for deformation prediction is about 1 voxel [4. The situation becomes more complex when the surgeon performs cuts, retractions, or resections [4] 6]. The last two necessarily involve a cut. Thus, the modelling of cuts and their effects is fundamental. The main difficulty 
associated with a cut is the discontinuity of matter displacement it involves. Indeed, FEM cannot handle such discontinuities directly.

We model the organs subjected to surgical cuts, retractions, and resections via the extended finite element method (XFEM). This powerful method was introduced in 1999 by Moës et al [1] in "fracture mechanics". This field deals with the appearance of cracks, which should be viewed as material discontinuities, and with their progression in mechanical structures such as airplane wings. The hope is that XFEM will reduce computational and memory requirements. Thus, it may hold the key to real-time modelling of deformation in surgical simulation and navigation.

In Sect. 2, we discuss the difficulties encountered with FEM when dealing with cracks. In Sect. 3, we review the main methods used in fracture mechanics. In Sect. 4, we introduce the basic principles of XFEM. In Sect. 5, a preliminary 2D proof-of-concept example, based on real data, is provided for the modelling of brain tissue retraction. Sect. 6 holds the conclusions.

\section{Limitations of FEM for Modelling Cracks}

Consider a solid, such as an organ, modelled by a volume mesh of elements, typically tetrahedra. The FEM approximation of the displacement $\boldsymbol{u}(\boldsymbol{x})$ of any point $\boldsymbol{x}$ in this solid is defined by 3 ]

$$
\boldsymbol{u}(\boldsymbol{x})=\sum_{i=1}^{N} \varphi_{i}(\boldsymbol{x}) \boldsymbol{u}_{i},
$$

where $i$ is the node index, $N$ the number of nodes in the mesh, $\boldsymbol{u}_{i}$ the displacement of node $i$, and $\varphi_{i}(\boldsymbol{x})$ the nodal shape function (NSF) with compact nodal support defined by the space occupied by all elements connected to node $i$. The $\boldsymbol{u}_{i}$ 's are also referred to as the nodal degrees of freedom (DOF): these are the discrete unknowns solved for in the FEM computation. The NSFs $\varphi_{i}(\boldsymbol{x})$ of FEM are defined to be continuous on each element. Thus, FEM has no built-in way of handling a crack going through an element. The only solution is remeshing, which involves the addition of nodes and elements [9] or topology adaptation [7], but these operations are computationally expensive. This makes FEM unsuitable for efficient crack modelling.

\section{Methods for Modelling Cracks}

Currently, there are three main methods for avoiding the drawbacks of FEM remeshing [10]. The boundary element method (BEM) dates back to the 60's and reached its peak of popularity in the 80 's. Originally, it was not designed or used for modelling cracks. BEM is based on the discretization of only the object surface. The size of the corresponding set of equations is thus greatly reduced. However, in contrast to FEM, these equations are non-symmetric 
and fully populated. BEM takes advantage of the fact that surface meshing is generally easier than volume meshing [13. When a crack appears and grows, new boundary elements must be added only along the crack. So BEM can avoid much of the remeshing required by FEM. BEM was used to study deformation due to brain shift, thus without any tissue discontinuity [1].

Meshless methods appeared in the 70's but it is only since the 90's that they have received significant attention [15. Their goal is to address large deformations and cracks 14. In FEM, the object is represented by a volume mesh. The nodes interact because they are connected via the elements. In meshless methods, the object is represented by a set of non-connected nodes that interact because their NSFs overlap. To model a crack, one cancels the interaction between some nodes by limiting the influence domain of their NSFs. While remeshing is avoided, the computation of the NSFs and the high-order Gauss quadrature required to compute the deformation equations can lead to greater computational requirements than for FEM [3. Nevertheless, meshless methods are useful when a problem benefits from being solved with a displacement approximation that does not rely on mesh topology [3. Meshless methods have been used to develop a surgical simulator [16 and to model the often-significant deformations of a biomechanical model of the beating heart [12].

XFEM was developed in 1999 specifically for studying cracks [1]. First, an FEM model, i.e., a mesh and associated NSFs, is built while ignoring the crack. Then, based upon the precise geometry of the crack, simple, auxiliary NSFs are added to some of the existing nodes. The solution of the equations can thus naturally provide a discontinuity in displacement at all points along the crack. The main appeal of XFEM is that it can model, without any remeshing, the deformations due to cracks of arbitrary shapes and also the way they propagate through matter. The equations remain also sparse and symmetric. Since XFEM can be viewed as an extension of FEM, one should be able to add XFEM capabilities to existing FEM frameworks.

\section{Introduction to Basic XFEM Principles}

XFEM works by allowing the solution of its equations to be discontinuous within mesh elements. Arbitrarily-shaped cracks can then be modelled without any remeshing. To provide a discontinuous solution, the displacement approximation $\boldsymbol{u}(\boldsymbol{x})$ of Eq. (11) should be expressed, not only in terms of the continuous NSFs $\varphi_{i}(\boldsymbol{x})$ of FEM, but also in terms of some auxiliary discontinuous NSFs. The key idea of XFEM is to "enrich" the nodes whose support is fully or partially intersected by the crack: one says that the support contains a "crack interior" and a "crack tip", respectively. Enrichment is performed by adding DOFs and associated auxiliary NSFs, which are the NSFs $\varphi_{i}(\boldsymbol{x})$ multiplied by some enrichment function (EF). 
If the crack fully intersects the support of a node, this node is enriched with the crack-interior EF given by the Heaviside function, a piecewise-constant function that changes sign at the crack boundary $\Gamma_{d}$, i.e.,

$$
H(\boldsymbol{x})=\left\{\begin{array}{rll}
1 & \text { for } & \left(\boldsymbol{x}-\boldsymbol{x}^{*}\right) \cdot \boldsymbol{e}_{n}>0 \\
-1 & \text { for } & \left(\boldsymbol{x}-\boldsymbol{x}^{*}\right) \cdot \boldsymbol{e}_{n}<0
\end{array}\right.
$$

where $\boldsymbol{x}$ is a point of the solid, $\boldsymbol{x}^{*}$ the point on $\Gamma_{d}$ closest to $\boldsymbol{x}$, and $\boldsymbol{e}_{n}$ the unit outward normal 1 to $\Gamma_{d}$ at $\boldsymbol{x}^{*} . H(\boldsymbol{x})$ cannot be used when $\Gamma_{d}$ does not fully intersect the support, because one would then effectively extend the discontinuity to the boundary of the support.

Consequently, nodes that have a crack tip within their support are enriched with specific crack-tip EFs that incorporate the radial and angular behavior of the asymptotic crack-tip displacement field, two-dimensional by nature. For an isotropic elastic material, the crack-tip EFs ar£

$$
\left\{F_{l}(r, \theta)\right\}_{l=1}^{4}=\left\{\sqrt{r} \sin \left(\frac{\theta}{2}\right), \sqrt{r} \cos \left(\frac{\theta}{2}\right), \sqrt{r} \sin \left(\frac{\theta}{2}\right) \sin (\theta), \sqrt{r} \cos \left(\frac{\theta}{2}\right) \sin (\theta)\right\},
$$

where $r$ and $\theta$ are the local polar coordinates. These crack-tip EFs ensure that the crack terminates precisely at the location of the crack tip and that the model possesses a correct near-tip behavior.

The XFEM approximation for a single crack with a single crack tip is thus

$$
\boldsymbol{u}(\boldsymbol{x})=\sum_{i \in I} \varphi_{i}(\boldsymbol{x}) \boldsymbol{u}_{i}+\sum_{j \in J} \varphi_{j}(\boldsymbol{x}) H(\boldsymbol{x}) \boldsymbol{a}_{j}+\sum_{k \in K} \varphi_{k}(\boldsymbol{x})\left(\sum_{l=1}^{4} F_{l}(\boldsymbol{x}) \boldsymbol{c}_{k}^{l}\right),
$$

where the $\boldsymbol{u}_{i}$ 's are the nodal degrees of freedom (DOFs) associated with the continuous part of the FE solution, the $\boldsymbol{a}_{j}$ 's the nodal enriched DOFs associated with the crack-interior EF, and the $c_{k}^{l}$ 's the nodal enriched DOFs associated with the crack-tip EFs. $I$ is the set of all nodes in the mesh, $J$ the set of nodes with supports cut by the crack interior, and $K$ the set of nodes with supports cut by the crack tip (Fig. 1). Equation (4) can be generalized to several cracks and crack tips. For details regarding the theory and various implementation issues, refer, e.g., to [1] 2] 3], and particularly to [2] for equations.

\section{Proof-of-Concept Example: Simulation of 2D Retraction}

To evaluate the capabilities and potential of XFEM for surgical simulation and navigation, we have performed preliminary tests on $2 \mathrm{D}$ objects containing a line-segment crack. The test program was written in MATLAB.

\footnotetext{
1 "Outward" is defined in an obvious way based upon the relative positions of $\boldsymbol{x}$ and $\Gamma_{d}$.

${ }^{2}$ The first function is discontinuous at the surface of the crack.
} 




Fig. 1. The mesh and crack geometries are respectively in gray and black. The nodes enriched with the crack-interior EF are represented by circles (set J) while nodes enriched with the crack-tip EFs are represented by squares (set K).

The inputs to the program are the mesh definition and the crack geometry. One begins by identifying the mesh elements that are fully intersected by the crack and the mesh elements that contain a crack tip. One defines the number of DOFs for each node: 2 for a non-enriched node, 4 for a node enriched with a crack-interior EF, or 10 for a node enriched with crack-tip EFs. As with FEM, each elementary stiffness matrix is computed, taking into account the EFs, and the global stiffness matrix is subsequently assembled. Force or displacement constraints are applied in similar ways in both FEM and XFEM.

We conducted a series of experiments. First, we used simple geometric shapes. The triangular mesh was created using the MATLAB PDE toolbox. Our first published example can be found in [18]. Then, we used realistic inputs. In the present case, we start from a segmented 3D image of the cortex and select one of its horizontal slice (Fig. 2(a)). The segmentation is performed as described in 8 (Fig. 2(b)). The mesh is computed using Distmesh2D 3 (Fig. 2(c)). The linear crack is shown in all images of Fig. 2. It starts at the brain surface and finishes close to the tumor, defining an incision segment (planar facet in 3D). To simulate the effect of a retractor (2D) that spreads out tissue from the incision segment, we compute the points of intersection of the crack with the mesh. Then, we impose an arbitrary displacement of $(-3.5,-1)$ and $(+3.5,+1)^{4}$ to each intersection, with the mesh, of the left and right crack lips, respectively. The intersection of the crack with the element containing the crack tip was left free to avoid constraints that are too large near the tip. Indeed, we have noticed that element flip causing element overlap can sometimes happen in this situation.

The application of this displacement constraint in XFEM is straightforward. For the intersection $\left(\boldsymbol{x}_{\text {int }}\right)$ of the crack with an element defined by 3 nodes enriched with $H(\boldsymbol{x})$, the relations from (4) between the nodal DOF 5 are

\footnotetext{
${ }^{3}$ Download from http://www-math.mit.edu/ persson/mesh/.

${ }^{4}$ The 1 st coordinate is vertical and the 2 nd horizontal (with reference to Fig. 2 .

${ }^{5}$ We consider the intersection lying on the element boundary between node 1 with DOFs $\left(u_{x 1}, u_{y 1}, a_{x 1}, a_{y 1}\right)$ and node 2 with DOFs $\left(u_{x 2}, u_{y 2}, a_{x 2}, a_{y 2}\right)$.
} 


$$
\left\{\begin{array}{l}
\varphi_{1}\left(\boldsymbol{x}_{i n t}\right) u_{x 1}+\varphi_{1}\left(\boldsymbol{x}_{i n t}\right) a_{x 1}+\varphi_{2}\left(\boldsymbol{x}_{i n t}\right) u_{x 2}+\varphi_{2}\left(\boldsymbol{x}_{i n t}\right) a_{x 2}=-3.5 \\
\varphi_{1}\left(\boldsymbol{x}_{i n t}\right) u_{y 1}+\varphi_{1}\left(\boldsymbol{x}_{i n t}\right) a_{y 1}+\varphi_{2}\left(\boldsymbol{x}_{i n t}\right) u_{y 2}+\varphi_{2}\left(\boldsymbol{x}_{i n t}\right) a_{y 2}=-1
\end{array}\right.
$$

for the left lip, where $H(\boldsymbol{x})$ is equal to +1 . Similarly, we have

$$
\left\{\begin{array}{l}
\varphi_{1}\left(\boldsymbol{x}_{i n t}\right) u_{x 1}-\varphi_{1}\left(\boldsymbol{x}_{i n t}\right) a_{x 1}+\varphi_{2}\left(\boldsymbol{x}_{i n t}\right) u_{x 2}-\varphi_{2}\left(\boldsymbol{x}_{i n t}\right) a_{x 2}=3.5 \\
\varphi_{1}\left(\boldsymbol{x}_{i n t}\right) u_{y 1}-\varphi_{1}\left(\boldsymbol{x}_{i n t}\right) a_{y 1}+\varphi_{2}\left(\boldsymbol{x}_{i n t}\right) u_{y 2}-\varphi_{2}\left(\boldsymbol{x}_{i n t}\right) a_{y 2}=1
\end{array}\right.
$$

for the right lip, where $H(\boldsymbol{x})$ is equal to -1 . Finally the discrete displacement solution provided by XFEM calculation was used to warp the brain image according to Eq. (4). The result of warping the image of Fig. [2(a) masked with the region of Fig. 2(b) is shown in Fig. 2(d).

If one attempts to use standard FEM to cope with a material discontinuity, the computation time will increase significantly. Indeed, one must modify the mesh in the vicinity of the discontinuity for the boundaries of the new elements to be aligned with the discontinuity. The nodes on the discontinuity must be identified and duplicated. The matrices representing the system of equations must be updated to take into account the new connectivity. In contrast, XFEM does not require remeshing. However, there are some costs associated with XFEM. While XFEM requires identification of the elements intersected by the crack and the use of $H(\boldsymbol{x})$ requires some geometrical calculations, the primary cost is associated with the calculation of the stiffness matrix of the elements containing the nodes that are enriched. The dimension of elementary stiffness matrices increase from $6 \times 6$ for a triangular element with 3 non-enriched nodes to $30 \times 30$ for a triangular element with 3 nodes enriched by crack-tip EFs. In contrast, FEM always requires a $6 \times 6$ stiffness matrix. The stiffness matrix computation for an element including a node enriched with crack-tip EFs involves analytical computation of their derivatives and Gauss quadrature to numerically integrate over the element.

\section{Conclusions}

Being able to model the deformations of organs is important both for surgical simulations and for image-guided navigation. This problem becomes complex and computationally intensive if one wishes to model the effects of cuts, retractions, and resections on the deformations of these organs. Current approaches that use a biomechanical model together with FEM to compute deformation require an expensive remeshing as soon as the volume mesh is cut.

This paper introduces a new approach that totally avoids the need for remeshing. In contrast with FEM, the solution provided by XFEM can now contain discontinuities of arbitrary shape inside mesh elements. Since the underlying framework remains that of FEM, the equations remain sparse and symmetric, thereby maintaining the computational efficiency of FEM. Of course, the number of DOFs increases in the case of XFEM because of the node enrichment procedure. Since XFEM can be viewed as an extension of 


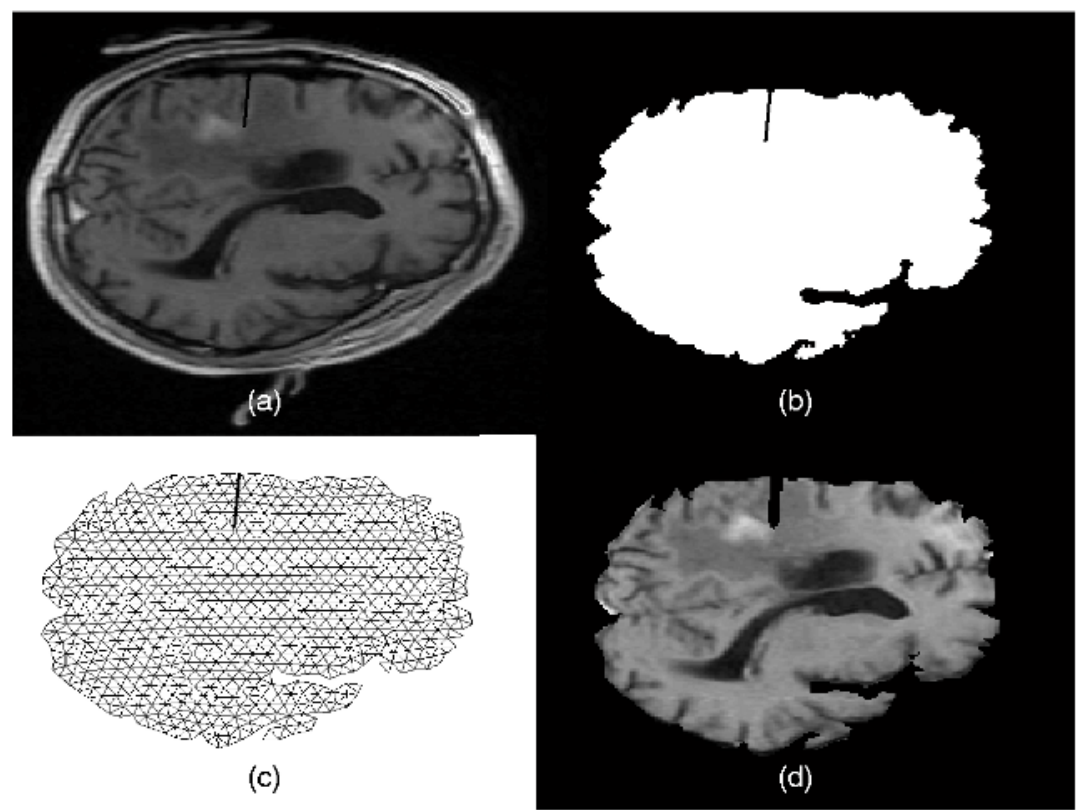

Fig. 2. (a) Original 2D MRI image with cut (discontinuity) leading from top surface to tumor. (b) Binary image of the cortex extracted from (a). (c) Triangular mesh computed from (b). (d) Image showing the result of deforming (a), masked with the region of (b), as specified by the retraction simulation described in the text.

FEM, XFEM techniques should fit well with existing FEM-based codes and applications.

The example of Section 5 confirms that XFEM can elegantly and efficiently take into account displacement discontinuities in the study of the mechanical properties of objects. This and other initial experiments we have performed give us confidence that XFEM may become a key tool for surgical simulation and image-guided navigation. In subsequent work, we will validate the method.

Acknowledgements. This work was sponsored in part by the "Fonds National de la Recherche Scientifique (F.N.R.S.)," Brussels, Belgium, the "Fonds Spéciaux pour la Recherche," University of Liège, Belgium, the "Léon Fredericq Foundation," University of Liège, Belgium, the Whitaker Foundation and by NIH grants R21 MH67054, R01 LM007861, P41 RR13218 and P01 CA67165. 


\section{References}

1. N. Moës, J. Dolbow, T. Belytschko: A finite element method for crack growth without remeshing. Int. J. Numer. Meth. Engng., 46: 131-150 (1999)

2. N. Sukumar, J.-H. Prévost: Modeling Quasi-Static Crack Growth with the Extended Finite Element Method. Part I: Computer Implementation. Internat. J. Solids Structures, 40(26): 7513-7537 (2003)

3. J.E. Dolbow: An Extended Finite Element Method with Discontinuous Enrichment for Applied Mechanics. PhD Dissertation, Northwestern University (1999)

4. M. Ferrant, A. Nabavi, B. Macq, R. Kikinis, S. Warfield: Serial registration of intra-operative MR images of the brain. Med. Image Anal., 6: 337-359 (2002)

5. J.G. Verly, L.M. Vigneron, N. Petitjean, C. Martin, M. Hogge, J. Mercenier, V. Jamoye, P.A. Robe: Nonrigid registration and multimodality image fusion for 3D image-guided neurochirurgical planning and navigation. Medical Imaging 2004, SPIE Proc. 5367 (Feb 2004)

6. M.I. Miga, D.W. Roberts, F.E. Kennedy, L.A. Platenik, A. Hartov, K.E. Lunn, K.D. Paulsen: Modeling of Retraction and Resection for Intraoperative Updating of Images. Neurosurgery, 49(1): 75-84 (2001)

7. D. Serby, M. Harders, G. Székely: A New Approach to Cutting into Finite Element Models. MICCAI'01, 425-433 (2001)

8. S.K. Warfield, M. Kaus, F.A. Jolesz, R. Kikinis: Adaptive, Template Moderated, Spatially Varying Statistical Classification. Med. Image Anal., 4(1): 43-55 (2000)

9. H.-W. Nienhuys: Cutting in deformable objects. PhD thesis, Institute for Information and Computing Sciences, Utrecht University (2003)

10. M. Duflot, H. Nguyen-Dang: A meshless method with enriched weight functions for fatigue crack growth. Int. J. Numer. Meth. Engng., 59: 1945-1961 (2004)

11. O. Ecabert, T. Butz, A. Nabavi, J.-P. Thiran: Brain Shift Correction Based on a Boundary Element Biomechanical Model with Different Material Properties. MICCAI'03, 2878: 41-49 (2003)

12. H. Liu and P. Shi: Meshfree Representation and Computation: Applications to Cardiac Motion Analysis. IPMI, 560-572 (July 2003)

13. J.O. Watson: Boundary Elements from 1960 to the Present Day. Electronic Journal of Boundary Elements, 1(1): 34-46 (2003)

14. T. Belytschko, Y. Krongauz, D. Organ, M. Fleming, and P. Krysl: Meshless methods: An overview and recent developments. Comput. Methods Appl. Mech. Engng., 139: 3-47 (1996)

15. G.R. Liu: Mesh Free Methods: Moving Beyond the Finite Element Method. CRC Press (2002)

16. S. De, J.W. Hong, K.J. Bathe: On the method of finite spheres in applications: towards the use with ADINA and in a surgical simulator. Comp. Mech., 31: 27-37 (2003)

17. P.-O. Persson, G. Strang: A Simple Mesh Generator in MATLAB. SIAM Review 46(2): 329-345 (2004)

18. L. Vigneron, J. Verly, S. Warfield: On Extended Finite Element Method (XFEM) for modelling of organ deformations associated with surgical cuts. Second International Symposium on Medical Simulation (June 2004) 